\title{
Co-infection of Coronavirus Disease 2019 and Influenza A: A Report from Iran
}

\author{
Zohre Khodamoradi, MD, MPH${ }^{1,2}$; Mohsen Moghadami, MD³ ${ }^{3 *}$ Mehrzad Lotfi, MD $^{4}$ \\ ${ }^{1}$ Department of internal medicine, Namazee Hospital, Shiraz University of Medical Sciences, Shiraz, Iran \\ ${ }^{2}$ Shiraz Geriatric Research Center, Shiraz University of Medical Sciences, Shiraz, Iran \\ ${ }^{3}$ Health Policy Research Center, Institute of Health, Shiraz University of Medical Sciences, Shiraz, Iran \\ ${ }^{4}$ Department of Radiology, Namazee Hospital, Shiraz University of Medical Sciences, Shiraz, Iran
}

\begin{abstract}
Background: In late December 2019, a viral pneumonia known as coronavirus disease 2019 (COVID-19) originated from China and spread very rapidly in the world. Since then, COVID-19 has become a global concern and health problem.

Methods: We present four patients in this study, selected from among patients who presented with pneumonia symptoms and were suspicious for COVID-19. They were referred to the intended centers for COVID-19 diagnosis and management of Shiraz University of Medical Sciences in southern Iran. Two nasopharyngeal and oropharyngeal throat swab samples were collected from each patient and tested for severe acute respiratory syndrome coronavirus 2 (SARS-CoV-2) using real-time reverse-transcriptasepolymerase-chain-reaction (RT-PCR). The samples were also tested for influenza viruses and the complete respiratory panel. Results: In the present report, four patients were diagnosed in the starting days of COVID-19 disease in our center in southern Iran with co-infection of SARS-CoV-2 and influenza A virus.

Conclusion: This co-infection of COVID-19 and influenza A highlights the importance of considering SARS-CoV-2 PCR assay regardless of other positive findings for other pathogens in the primary test during the epidemic.

Keywords: Coinfection, Coronavirus disease 2019, COVID-19, Influenza, Severe acute respiratory syndrome coronavirus 2

Cite this article as: Khodamoradi Z, Moghadami M, Lotfi M. Co-infection of coronavirus disease 2019 and influenza a: a report from Iran. Arch Iran Med. 2020;23(4):239-243. doi: 10.34172/aim.2020.04.
\end{abstract}

Received: March 20, 2020, Accepted: March 21, 2020, ePublished: April 1, 2020

\section{Introduction}

Coronaviruses are enveloped RNA viruses from the Coronaviridae family, which can spread to humans and other mammals. ${ }^{1-2}$ Two epidemics of betacoronaviruses have occurred in the world with typical characteristics of a respiratory syndrome, which were named as severe acute respiratory syndrome coronavirus (SARS-CoV) and Middle East respiratory syndrome coronavirus (MERS$\mathrm{CoV}$ ) in the $21^{\text {st }}$ century. The overall mortality rates of these two epidemics of SARS-CoV and MERS-CoV were $10 \%$ and $37 \%$, respectively. $3-6$

A new betacoronaviruses from the Coronaviridae family, which first originated and was detected in Wuhan, Hubei, China, in December 2019, caused a cluster of viral pneumonia cases. ${ }^{2}$ The first confirmed case was recognized in the samples of the bronchoalveolar lavage fluid of a patient in Wuhan on January 3, 2020. This viral pneumonia is known as coronavirus disease 2019 (COVID-19) and has spread very rapidly in the world with cases detected in multiple countries. ${ }^{13,5,7}$ About 242209 confirmed infected cases have been detected, and 9992 deaths have been reported in the world as of March 19, 2020, most of them from China. The number of infected cases and involved countries increases daily; thus,
COVID-19 has become a global public health emergency. One hundred thirty-seven countries have experienced COVID-19 up to now. ${ }^{8}$ Moreover, COVID-19 can kill healthy adults.

Most of the patients infected with COVID-19 in the world presented with fever, fatigue, dry cough, and dyspnea. In addition to these symptoms, some cases were reported with gastrointestinal manifestations such as diarrhea and nausea as the initial presentation of COVID-19. ${ }^{1,6,9-10}$ As reported in previous studies, the most common finding in lung computed tomography (CT) scan is bilateral multifocal ground-glass opacities, a crazy-paving pattern, and consolidation, which is a typical finding in viral pneumonia. These clinical manifestations are similar to what was found in SARS-CoV and MERS$\mathrm{CoV}$ patients. ${ }^{6,9,11-12}$

The most common laboratory findings on admission are lymphopenia, thrombocytopenia, leukopenia, and elevated C-reactive protein. Moreover, high levels of alanine aminotransferase, and aspartate aminotransferase have been reported. ${ }^{6}$

This report aims to describe four Iranian cases infected simultaneously with COVID-19 and influenza A at the time of referral to the emergency room. We report the 
diagnostic protocol, clinical manifestations, laboratory data, and radiological findings of these patients with confirmed COVID-19 infection.

\section{Material and Methods}

Diagnostic and Confirmatory Test

The genetic sequence of 2019-nCoV was shared by Chinese researchers on January 7, 2020. ${ }^{13}$ Patients who are suspected cases of COVID-19 should undergo testing for COVID-19 using the real-time reversetranscriptase-polymerase-chain-reaction (RT-PCR) assay. Two nasopharyngeal and oropharyngeal throat swab samples were collected and tested for SARS-CoV-2 for each patient using the Chinese Center for Disease Control and Prevention (CDC) recommended Kit (BioGerm, Shanghai, China), following the WHO guidelines for RTPCR. ${ }^{14,15}$

According to the protocol of COVID-19 detection in suspicious patients, samples also should be tested for influenza viruses and complete respiratory panels for detection of parainfluenza virus, adenovirus, human rhinovirus, respiratory syncytial virus, Bordetella pertussis, Chlamydia pneumoniae, and Mycoplasma pneumonia.

\section{Patients}

The cases presented in this study were patients with confirmed samples of COVID-19 and influenza A. The samples were collected from patients who presented with pneumonia symptoms and were suspicious for COVID-19; they referred to the intended centers for COVID-19 diagnosis and management of Shiraz University of Medical Sciences in southern Iran.

\section{Data Gathering}

The data were gathered by reviewing the patients' medical records, and the documented laboratory data were then submitted in prepared datasheets. The patients' demographic information, including age and gender, was recorded, as well as the patients' clinical presentations on admission.

\section{Case Presentation}

Four patients presented with pneumonia and were admitted to a hospital which is the center for COVID-19 diagnosis and management of Shiraz University of Medical Sciences.

On February 24, 2020, a 74-year-old woman (patient 1 ), who was a case of previous ischemic cerebrovascular accident and hypertension referred to the emergency department of our local center for COVID-19 diagnosis and management of Shiraz University of Medical Sciences. She had a history of five days of dry cough, malaise, body pain, subjective fever, headache, and anorexia. She had developed dyspnea and orthopnea one day before admission. She did not report any history of gastrointestinal symptoms. She had no contact with confirmed COVID-19 patients but had contact with patients with cough in a clinic when she had referred to the physician for her routine checkup.

On admission, physical examination revealed a blood pressure of $70 / 50 \mathrm{~mm} \mathrm{Hg}$, body temperature of $38.7^{\circ} \mathrm{C}$, pulse rate of 89 beats per minute, respiratory rate of 26 breaths per minute, and oxygen saturation of $90 \%$, while the patient was breathing ambient air.

Her chest radiography on the first day of admission showed diffuse infiltrates in both lungs (Figure 1).

Her swab sample for was positive the influenza A viruses. We sent other nasopharyngeal and oropharyngeal samples which were positive for SARS-CoV-2 on RT-PCR assays. This positive test for SARS-CoV-2 confirmed the diagnosis of COVID-19 in this patient, but the respiratory panel was negative.

The second patient was a 40-year-old healthy man who had travelled to a city involved with COVID-19 with a high number of confirmed cases (Qom) before the onset of his symptoms. Moreover, he stated that he had close contact with his friends who lived in that city. His symptoms started during his stay. Four days after his travel to that city, his symptoms initiated with headache and fever. This implies that the incubation period for COVID-19 may have been three days in this case. About 8 hours later, his initial symptoms progressed, and he developed sweating, chills, cough, and severe compressive chest pain. On the second day of illness, he presented with dyspnea, orthopnea, and body pain. In addition to his symptoms, he reported diarrhea on the second day of the disease which lasted for three days. His chest pain worsened, so he returned to Shiraz and was admitted to our local center for COVID-19 diagnosis and management on the fourth day, i.e. February 24, 2020. On admission, he was ill with respiratory distress, sweating, and a low-grade fever. He complained of very severe compressive chest pain, orthopnea, and body pain.

At the first visit by the physician in the emergency room, physical examination revealed a blood pressure of $110 / 70 \mathrm{~mm} \mathrm{Hg}$, body temperature of $35.4^{\circ} \mathrm{C}$, pulse rate of 77 beats per minute, respiratory rate of 20 breaths per minute, and oxygen saturation of $97 \%$, while the patient
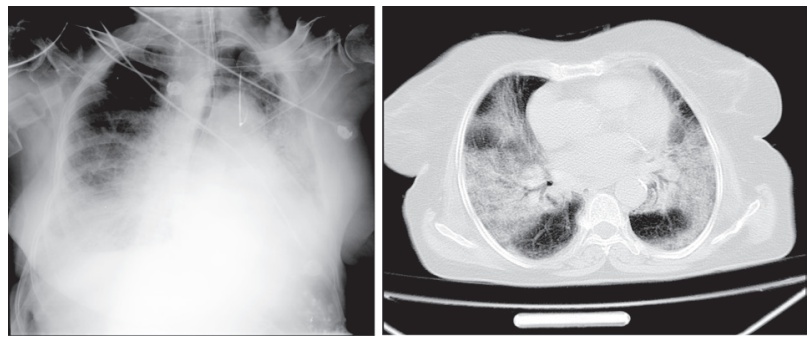

Figure 1. Sample Images of the Thoracic CT Scans of the Lungs and Posteroanteriorc Chest Radiography of Patient 1. 
was breathing ambient air.

His chest radiography showed diffuse and bilateral infiltration in the lungs (Figure 2). Similar to patient No. 1 , his swab sample for was positive the influenza A viruses, and samples for SARS-CoV-2 on RT-PCR assays were positive, as well. Thus, the second case of co-infection of influenza A with COVID-19 was found.

On February 25, 2020, a 64-year-old man presented to our local center for COVID-19 diagnosis and management with a complaint of dry cough, malaise, headache, and subjective fever for about five days. Additionally, he reported that he had dyspnea for a few hours. He did not report any gastrointestinal symptoms or chest pain. On admission, physical examination revealed a blood pressure of $130 / 80 \mathrm{~mm} \mathrm{Hg}$, body temperature of $37.7^{\circ} \mathrm{C}$, pulse rate of 110 beats per minute, respiratory rate of 19 breaths per minute, and oxygen saturation of $87 \%$, while he was breathing ambient air. Chest radiography taken on the first day of admission was similar to what was found in previous patients; diffuse and bilateral infiltration was detected in the lungs (Figure 3). Following our protocol for all patients suspicious for COVID-19, rapid nucleic acid amplification test for influenza $\mathrm{A}$ and $\mathrm{B}$ and respiratory panel were performed and his influenza A report was positive. Nasopharyngeal and oropharyngeal swab specimens were positive for SARS-CoV-2 on RTPCR assays. He was the third patient with positive influenza A and COVID-19 on admission in our center for COVID-19 diagnosis and management affiliated to

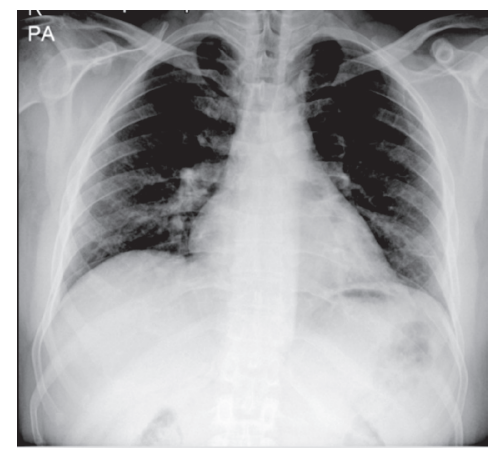

Figure 2. Posteroanterior Chest Radiography of Patient 2.
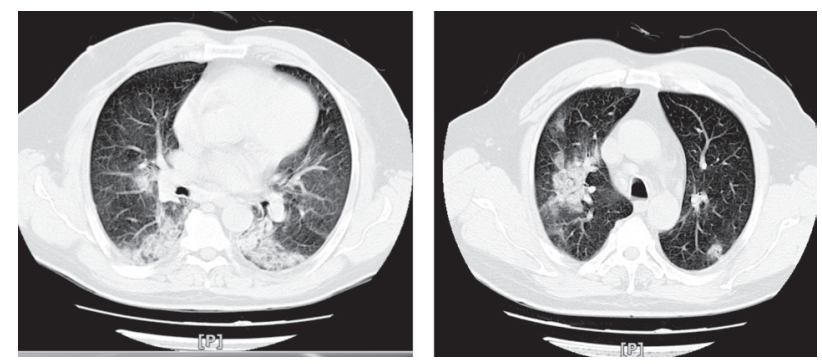

Figure 3. Sample Images of the Thoracic CT Scans of the Lungs and Posteroanterior Chest Radiography of Patients 3.
Shiraz University of Medical Sciences.

The fourth patient was a 50-year-old healthy man without any underlying disease who was referred to the center for COVID-19 diagnosis and management on March 2, 2020. He stated that he had travelled to Qom about two weeks before the onset of his symptoms. After he returned to Shiraz, he presented with subjective fever, dry cough, and dyspnea on February 30. His dyspnea progressed, so he was admitted to our center. On admission, blood pressure of $120 / 65 \mathrm{~mm} \mathrm{Hg}$ was found. He had body temperature of $38.0^{\circ} \mathrm{C}$, pulse rate of 85 beats per minute, respiratory rate of 18 breaths per minute, and oxygen saturation of $93 \%$, while the patient was breathing ambient air. His chest radiography on the first day of admission showed diffuse infiltrates in both lungs (Figure 4).

On March 4, his positive nasopharyngeal and oropharyngeal swabs that were tested for COVID-19 and influenza A confirmed that he had simultaneous coinfection with COVID-19 and influenza A.

All demographic characteristics, clinical features, and laboratory findings of the four patients are shown in Table 1.

\section{Discussion}

There are several reports indicating that viral interactions have some bearing on the course of infectious diseases. ${ }^{16,17}$ However, these interactions are relatively undefined in the process and pathogenesis of most viral diseases. In this field, the majority of investigations have focused on chronic viral infections such as HIV and hepatitis B and C. ${ }^{18,19}$ Due to the comparatively short duration of viral respiratory infections, the topic of co-infection might be less of a concern.

In the present report, in an investigation of the first twelve cases, four patients in the starting days of COVID-19 disease in our region in southern Iran were identified as co-infection of SARS-CoV-2 and Influenza A virus. We conducted this study to determine the role of various viruses in the etiology of acute viral respiratory diseases and suggest an empirical therapy for the respiratory tract infectious disease in patients who refer to the primary clinics. However, the exact role of co-infection
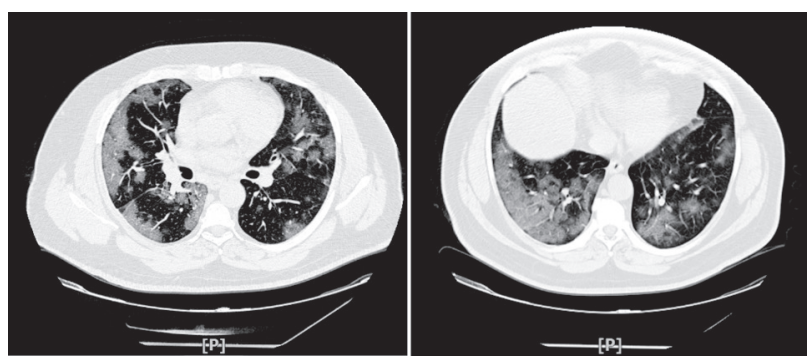

Figure 4. Sample Images of the Thoracic CT Scans of Lungs Patient 4. 
Table 1. Summary of Demographic Characteristics, Clinical Features, and Laboratory Findings of the 4 Patients Presenting with Co-infection of Coronavirus Disease 2019 and Influenza A

\begin{tabular}{|c|c|c|c|c|}
\hline Clinical Characteristics & Patient 1 & Patient 2 & Patient 3 & Patient 4 \\
\hline Date of admission & February 24 & February 24 & February 25 & March 2 \\
\hline Age & 74 & 40 & 64 & 50 \\
\hline Gender & Female & Male & Male & Male \\
\hline Underlying disease (risk factor) & Yes & No & No & No \\
\hline \multicolumn{5}{|l|}{ Sign and symptoms (on admission) } \\
\hline Cough & Yes & Yes & Yes & Yes \\
\hline Dyspnea & Yes & Yes & Yes & Yes \\
\hline Fever & Yes & Yes & Yes & Yes \\
\hline Body pain & Yes & Yes & No & Yes \\
\hline Chest pain & No & Yes & No & No \\
\hline Headache & Yes & Yes & Yes & No \\
\hline Sweating & No & Yes & No & No \\
\hline Diarrhea & No & Yes & No & No \\
\hline Nausea & No & Yes & No & No \\
\hline \multicolumn{5}{|l|}{ Laboratory data } \\
\hline White blood cell $\left(\times 10^{9}\right.$ cells per $\left.\mathrm{L}\right)$ & 4.3 & 4.1 & 6.2 & 4 \\
\hline Neutrophil count $\left(\times 10^{9}\right.$ cells per $\left.\mathrm{L}\right)$ & 3.8 & 1.6 & 3.9 & 3.1 \\
\hline Lymphocyte count $\left(\times 10^{9}\right.$ cells per $\left.\mathrm{L}\right)$ & 0.3 & 1.9 & 1.1 & 0.6 \\
\hline Platelet count $\left(\times 10^{9}\right.$ cells per $\left.\mathrm{L}\right)$ & 140 & 153 & 200 & 169 \\
\hline CRP (mg/L) & 24 & 10 & 45 & 55 \\
\hline $\operatorname{ESR}(\mathrm{mm} / \mathrm{h})$ & 69 & 7 & 51 & 44 \\
\hline Blood urea nitrogen $(\mathrm{mg} / \mathrm{dL})$ & 67 & 14 & 16 & 12 \\
\hline Creatinine $(\mathrm{mg} / \mathrm{dL})$ & 2.2 & 1.1 & 1.3 & 1.2 \\
\hline Aspartate aminotransferase (mg/dL) & 2220 & 25 & 56 & 171 \\
\hline Alanine aminotransferase (mg/dL) & 461 & 23 & 23 & 147 \\
\hline Radiologic abnormalities & Yes & Yes & Yes & Yes \\
\hline
\end{tabular}

CRP, C-reactive protein; ESR, erythrocyte sedimentation rate.

in the clinical course and whether these co-infections need improvement or change in the current treatment protocol are still unclear.

This is the first investigation that quantified the clinical severity of COVID-19 based on specific co-pathogens. Our co-infection rate of $33 \%$ is higher than what is reported in other studies. ${ }^{20,21}$ It might be due to the overlap between the emergence of SARS-CoV-2 and seasonal flu in our region. This overlap emphasizes the consideration of SARS-CoV-2 PCR test regardless of other positive findings for other pathogens in the primary test during the epidemic.

In our protocol, chest $\mathrm{CT}$ scan was the first line of screening and diagnosing COVID-19. Fang et al showed that chest CT scan has greater sensitivity than RT-PCR for detection of COVID-19. ${ }^{22}$ Ground glass opacities, multifocal patchy consolidation, especially with peripheral distribution, constitute the pattern of lung involvement in viral pneumonia and are non-specific for patients infected with COVID-19. Furthermore, this pattern is similar to the abnormality of the chest CT scan which was reported in the two previous coronavirus outbreaks of SARS and MERS. ${ }^{12,23-25}$ Therefore, these imaging findings guided us to find suspected patients by considering clinical findings. As mentioned, the four patients presented in this study had non-specific chest CT findings for viral pneumonia, which cannot differentiate influenza from COVID-19. Therefore, in this study, chest CT scan only helped us to find the viral infection in the lungs, while swab samples helped us to confirm influenza and COVID-19; then, we chose the proper management and therapeutic regimen accordingly.

\section{Authors' Contribution}

ZKh and MM designed the study, gathered all data, and co-wrote the paper. ML and MM supervised the project. All authors discussed the results and contributed to the final manuscript.

\section{Conflict of Interest Disclosures}

The authors declare that they have no conflict of interest.

\section{Ethical Statement}

The ethics committee of Shiraz University of Medical Sciences approved the study with the ethical protocol code: IR.SUMS. REC.1398.1377.

\section{Acknowledgements}

The authors would like to thank Manica Negahdaripour, the Shiraz University of Medical Sciences, Shiraz, Iran, the Center for Development of Clinical Research of Nemazee Hospital and Dr. Nasrin Shokrpour for editorial assistance.

\section{References}

1. Holshue ML, DeBolt C, Lindquist S, Lofy KH, Wiesman J, Bruce $\mathrm{H}$, et al. First case of 2019 novel coronavirus in the 
United States. N Engl J Med. 2020;382(10):929-36. doi: 10.1056/NEJMoa2001191.

2. Huang C, Wang Y, Li X, Ren L, Zhao J, Hu Y, et al. Clinical features of patients infected with 2019 novel coronavirus in Wuhan, China. Lancet. 2020;395(10223):497-506. doi: 10.1016/S0140-6736(20)30183-5.

3. Zhu N, Zhang D, Wang W, Li X, Yang B, Song J, et al. A novel coronavirus from patients with pneumonia in China, 2019. N Engl J Med. 2020;382(8):727-733. doi: 10.1056/ NEJMoa2001017.

4. Drosten C, Meyer B, Müller MA, Corman VM, Al-Masri $M$, Hossain R, et al. Transmission of MERS-coronavirus in household contacts. N Engl J Med. 2014;371(9):828-35. doi: 10.1056/NEJMoa1405858.

5. Wu JT, Leung K, Leung GM. Nowcasting and forecasting the potential domestic and international spread of the 2019-nCoV outbreak originating in Wuhan, China: a modelling study. Lancet. 2020;395(10225):689-697. doi: 10.1016/S01406736(20)30260-9.

6. Chowell G, Abdirizak F, Lee S, Lee J, Jung E, Nishiura H, et al. Transmission characteristics of MERS and SARS in the healthcare setting: a comparative study. BMC Med. 2015;13:210. doi: 10.1186/s12916-015-0450-0.

7. Hoehl S, Berger A, Kortenbusch M, Cinatl J, Bojkova D, Rabenau $\mathrm{H}$, et al. Evidence of SARS-CoV-2 Infection in Returning Travelers from Wuhan, China. N Engl J Med. 2020 Feb 18. doi: 10.1056/NEJMc2001899.

8. COVID-19 CORONAVIRUS OUTBREAK; 2020. Available from: https://www.worldometers.info/coronavirus/. Updated March 19, 2020; cited 2020 March 19, 2020.

9. Chan JF-W, Yuan S, Kok K-H, To KK-W, Chu H, Yang J, et al. A familial cluster of pneumonia associated with the 2019 novel coronavirus indicating person-to-person transmission: a study of a family cluster. Lancet. 2020;395(10223):514-23. doi: 10.1016/S0140-6736(20)30154-9.

10. Chen H, Guo J, Wang C, Luo F, Yu X, Zhang W, et al. Clinical characteristics and intrauterine vertical transmission potential of COVID-19 infection in nine pregnant women: a retrospective review of medical records. Lancet. 2020; 395(10226):809-815. doi: 10.1016/S0140-6736(20)30360-3.

11. Wang D, Hu B, Hu C, Zhu F, Liu X, Zhang J, et al. Clinical characteristics of 138 hospitalized patients with 2019 novel coronavirus-infected pneumonia in Wuhan, China. JAMA. 2020;323(11):1061-9. doi: 10.1001/jama.2020.1585.

12. Pan F, Ye T, Sun P, Gui S, Liang B, Li L, et al. Time course of lung changes on chest CT during recovery from 2019 novel coronavirus (COVID-19) pneumonia. Radiology. 2020:200370. doi: 10.1148/radiol.2020200370.

13. Zhou P, Yang XL, Wang XG, Hu B, Zhang L, Zhang W, et al. A pneumonia outbreak associated with a new coronavirus of probable bat origin. Nature. 2020;579:270-3. doi: 10.1038/ s41586-020-2012-7.

14. World Health Organization. Clinical management of severe acute respiratory infection when novel coronavirus (2019$\mathrm{nCoV}$ ) infection is suspected. Interim Guidance January. 2020; 28. Available from: https://www.who.int/publications-detail/ clinical-management-of-severe-acute-respiratory-infectionwhen-novel-coronavirus-(ncov)-infection-is-suspected.

15. Corman VM, Landt O, Kaiser M, Molenkamp R, Meijer A, Chu DK, et al. Detection of 2019 novel coronavirus (2019-nCoV) by real-time RT-PCR. Euro Surveill. 2020; 25(3):2000045. doi: 10.2807/1560-7917.ES.2020.25.3.2000045.

16. Pou AM, Rimell FL, Jordan JA, Barua P, Shoemaker DL, Post JC, et al. Adult Respiratory Papillomatosis: Human Papillomavirus Type and Viral Coinfections as Predictors of Prognosis. Los Angeles, CA: SAGE Publications Sage CA; 1995.

17. Franz A, Adams O, Willems R, Bonzel L, Neuhausen N, Schweizer-Krantz S, et al. Correlation of viral load of respiratory pathogens and co-infections with disease severity in children hospitalized for lower respiratory tract infection. J Clin Virol. 2010;48(4):239-45. doi: 10.1016/j.jcv.2010.05.007

18. Muriuki BM, Gicheru MM, Wachira D, Nyamache AK, Khamadi SA. Prevalence of hepatitis B and C viral co-infections among HIV-1 infected individuals in Nairobi, Kenya. BMC Res Notes. 2013;6(1):363. doi: doi.org/10.1186/1756-0500-6-363.

19. He N, Chen L, Lin H, Zhang M, Wei J, Yang J, et al. Multiple viral coinfections among HIV/AIDS patients in China. Bioscience Trends. 2011;5(1):1-9. doi: 10.5582/bst.2011.v5.1.1

20. Drews AL, Atmar RL, Glezen WP, Baxter BD, Piedra PA, Greenberg SB. Dual respiratory virus infections. Clin Infect Dis. 1997;25(6):1421-9.

21. Nisii C, Meschi S, Selleri M, Bordi L, Castilletti C, Valli MB, et al. Frequency of detection of upper respiratory tract viruses in patients tested for pandemic H1N1/09 viral infection. J Clin Microbiol. 2010;48(9):3383-5. doi: 10.1128/JCM.01179-10.

22. Fang $\mathrm{Y}$, Zhang $\mathrm{H}$, Xie J, Lin M, Ying L, Pang P, et al. Sensitivity of chest CT for COVID-19: comparison to RT-PCR. Radiology. 2020:200432. doi: doi.org/10.1148/radiol.2020200432

23. Bernheim A, Mei $X$, Huang $M$, Yang $Y$, Fayad ZA, Zhang $N$, et al. Chest CT Findings in Coronavirus Disease-19 (COVID-19): Relationship to Duration of Infection. Radiology. 2020:200463.doi: doi: 10.1148/radiol.2020200463

24. Ai T, Yang Z, Hou H, Zhan C, Chen C, Lv W, et al. Correlation of chest CT and RT-PCR testing in coronavirus disease 2019 (COVID-19) in China: a report of 1014 cases. Radiology. 2020:200642. doi: 10.1148/radiol.2020200642.

25. Kanne JP, Little BP, Chung JH, Elicker BM, Ketai LH. Essentials for radiologists on COVID-19: an update-Radiology Scientific Expert Panel. Radiology. 2020:200527. doi: 10.1148/radiol.2020200527. 